\section{Nervous System.}

Cranial Nerves. -The left pupil was smaller than the right, but both reacted to light and accommodation. Lateral nystagmus was present, which was more marked on looking to the right. The patient did not complain of diplopia and the ocular muscles acted perfectly. There was no weakness of any facial-muscles, nor did the tongue when protruded deviate to either side. All the special senses, including taste, were normal. The left side of the palate was paralysed, but Dr. D. Lindley Sewell found no affection of any of the laryngeal muscles, on kindly making a laryngoscopic examination for us. Analgesia and thermal anaesthesia were found over the greater part of the distribution of the ophthalmic and superior maxillary divisions of the left trigeminal nerve. The recognition of painful and thermal stimuli was defective over the left half of the palate. A small patch of herpes was present in the left malar region.

Scnsation.-In addition to the dissociated sensory loss on the upper part of the left side of the face, analgesia and thermal anaesthesia were found over the right half of the neck, trunk, and both extremities on this side. There was no disturbance of any other form of sensation. The precise extent of the dissociated sensory loss is represented in Fig. 1.
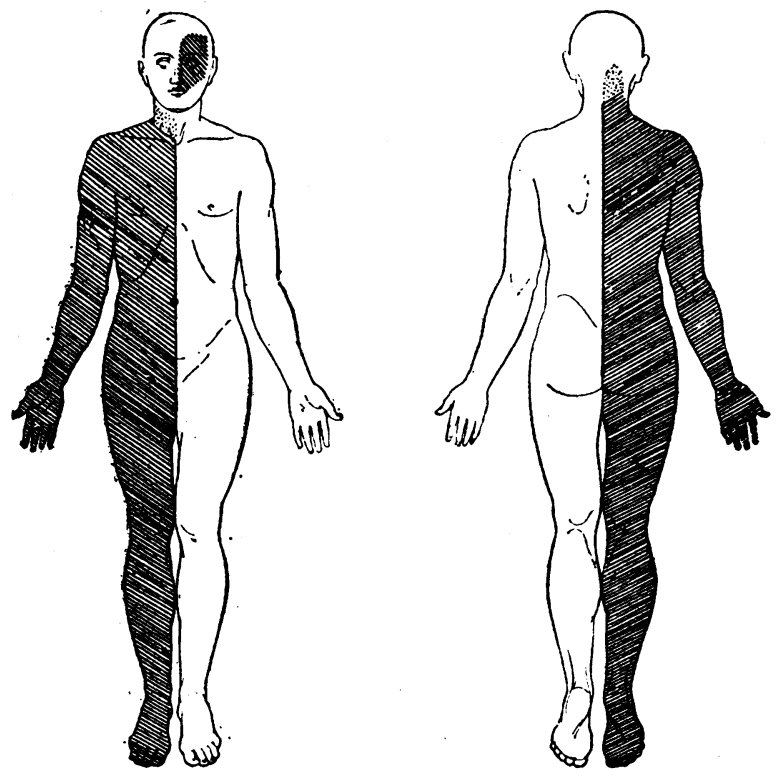

FIG. 1.-Showing the distribution of the analgesia and thermal anaesthesia.

Muscular System.-Movements of the left arm and leg exhibited marked ataxia, but no paralysis or paresis of any muscle could be discovered.

Rcflcrcs.-The reflexes of the superior extremity were normal The knee-jerks and $\Lambda$ chilles-jerks were present, but not very easily clieited. There was no ankle clonus and the plantar response was

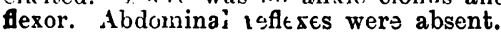

I'ne general examination revealed evidence of very general arterio-sclerosis, but the Wassermann test was negative. Albumin was present in the urine; the systolic blood pressure was 185 and the diastolic 120 . A blood urea estimation; kindly made by $\mathrm{Dr}$ G. E. Loveday, gave $76.0 \mathrm{mg}$. urea per $100 \mathrm{c.cm}$. of blood.

\section{After-History.}

During a stay in hospital of seven weeks the dysphagia disappeared, the ataxia became less marked so that the patient was able to walk about, and less difficulty was experienced in obtaining the knee-jerks, but the other manifestations persisted.

The clearly defined syndrome renders the anatomical localization of the lesion fairly simple (I'ig. 2). The sudden onset in a patient suffering from well marked arterial disease assists in arriving at a pathological diagnosis, but it is less easy to determine with certainty which vessel is affected, and some observers have frankly stated that it is impossible clinically to distinguish between occlusion of the vertebral and the posterior inferior cerebellar arteries. An anatomical stud $y^{1}$ has failed to solve this difficulty, but has provided the following information which may be of considerable value:

(a) Any paresis of the trunk, limb, or tongue muscles definitely denotes involvement of the vertebral or anterior spinal arteries (b) Crossed and dissociated senzory loss unassociated with dysphagia and laryngeal paralysis is most likely to be due to vertebral occlusion, since the spino-thalamic tract is never supplied by the posterior inferior cerebellar artery below the caudal extremity of the nucleus ambiguus.

(c) Rarely branches of the rertebral may replace the bulbar branches of the posterior inferior cerebellar, and occlusion of the former, at the point where these branches arise, may produce the characteristic syndrome which we associate with the latter without any paresis of trunk or limb muscles.
Three points of special interest are illustrated by this case. 1. Since the occluded vessel supplies a considerable part of the spinal tract and nucleus of the trigeminal nerve and never contributes any branches to the nuclei of this nerve in the pons, and since the loss of sensation on the same side of the face as the lesion is dissociated, it is believed that the spinal nucleus receives only pain and thermal fibres.

2. Anatomical investigation has proved that the more caudal part of the spinal nucleus and tract is most constantly supplied by the posterior inferior cerebellar artery, and that, when the bulbar branches of this vessel are reduced in number, it is the cephalic extremity which escapes. In the case reported in this paper it is significant that the sensory loss was confined to the upper part of the face, which confirms the opinion expressed by Spiller ${ }^{2}$ that the ophthalmic division of the fifth nerve descends to a lower level than the other two branches and that the fibres of the inferior maxillary division pass to the cephalic part of the nucleus.

3. On anatomical grounds it appears that in our patient the bulbar branches of the artery are less numerous than usual, which leads to a reduction in a cephalic direction of the retro-olivary region generally supplied. This results in

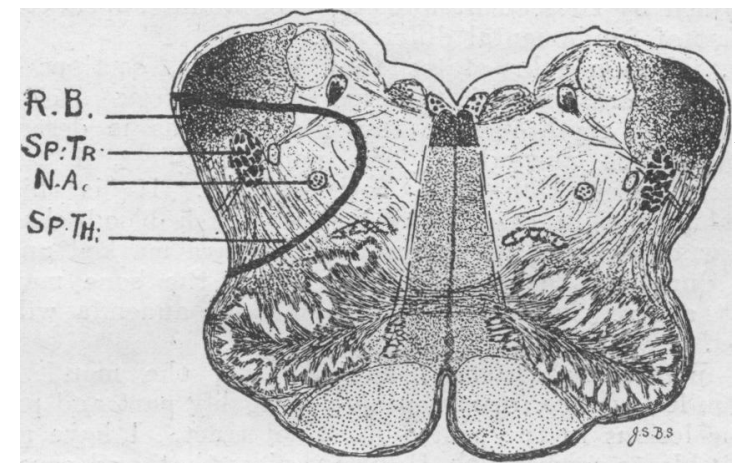

Fig. 2.-Section of the medulla at the mid-olivary level. The black line marks out the typical area of the meduila supplied by the bulbar branches of the posterior inferior cerebellar artery. R.B.=Restiform Nucleus ambiguus. Sp. Th.=Spino-thalamic tract.

escape of the upper part of the nucleus ambiguus as well as the cephalic extremity of the spinal nucleus and tract. Since no paralysis of larrngeal muscles was found, this ase supports the view that it is the upper part of the nucleus ambiguus which supplies fibres for the innervation of the muscles of the larynx, whilst the caudal part gives origin to the efferent fibres to the palate and striated muscles of the pharynx.

In conclusion, we desire to acinnowledge ou: indebtedness to Dr. A. Coleman, house-physician, for assistance in taking the notes. 1 Stopford, J. S. B.: The Arteries of the Pons and Medulla Oblongata.
Journ. of Anat., Part I, January, 1916, pp. 142-158; Part II, April, 1916,

pp. 255-280 ; Part III, April, 1917, pp. 250-277. Journ. of Nervous and Mental Disease, vol. xlii, 1915, pp. 399-418.

\section{SPRUE AND PERNICIOUS ANAEMIA. BY}

Sir BYROM BRAMWEJL, M.D., F.R.C.P., CONSULTING PHYSICIAN, EDINBURGH ROYAL INFIRMARY.

I READ Dr. J. L. Priston's communication, published in the Journal of February 2nd, with much interest. In this country we rarely see cases of sprue; but two very marked cases, both of which proved fatal, came under my observation in the years 1895 and 1899, and I was much impressed with the close resemblance between some cases of sprue and pernicious anaemia. In pernicious anaemia, glossitis and gastro-intestinal symptoms, which are such prominent features of sprue, are often present. In sprue, anaemia may be very marked, and, as Dr. Priston points out, in the advanced stages of that disease may present all the characteristic features of pernicious anaemia (high colour index, poikilocytosis, megalocytosis, etc.). Dr. Philip $\mathbf{H}$. Manson-Bahr, in his article on sprue in Byam and 
Archibald's Practice of Medicine in the Tropics (vol. iii, p. 2257), describes the condition of the blood in sprue in detail.

In sprue, as in pernicious anaemia, the profound anaemia appears to be due to the absorption of some blood-destroying toxin from the gastro-intestinal tract, probably a different toxin in the two diseases. In both diseases the blood destruction seems to take place chiefly in the portal circulation, with resulting deposits of pigment in the liver. In pernicious anaemia there is not the extreme emaciation of fat and muscle which is present in the advanced stages of sprue. In sprue the sudden remissions which are so often seen in pernicious anaemia are said not to occur; but this point requires, I think, further observation and corroboration.

Dr. A. F. Hurst has recently pointed out (BRITIsH Medical Journal, January 19th, 1924) that in pernicious anaemia there is no free hydrochloric acid in the stomach; while Dr. Priston states that in sprue the gastric contents may show hyperchlorhydria, normal acidity, or hypochlorhydria; this (that is, that in some cases of sprue in which the blood changes are characteristic of pernicious anaemia there is hyperchlorhydria or normal acidity), if confirmed by subsequent observations, would appear to be a point of fundamental difference.

Dr. Manson-Bahr states that in sprue, sections and other preparations of the red bone marrow show no evidence of erythroblastic response, such as is described in pernicious anaemia. This point requires, I think, further observation and corroboration. It is highly probable that in cases of sprue, in which blood changes similar to those of pernicious anaemia are marked and of long duration, the same alterations in the bone marrow which are so characteristic of pernicious anaemia will be found.

In pernicious anaemia, as in sprue, the mouth and tongue lesions are, in my opinion, probably part and parcel of the lesions in the gastro-intestinal tract. I have never been able to agree with Hunter's view that oral sepsis is the cause of pernicious anaemia. As I have more than once pointed out, I have met with cases of profound pernicious anaemia in which the patient for years before the onset of the disease had had no teeth, and in which the gums were perfectly healthy and there was not, and never had been, any source of oral sepsis. In sprue, even when the anaemia is very marked, it may not present the characteristic features of pernicious anaemia.

In my first typical and marked case of sprue, "the patient was extremely debilitated, greatly emaciated, and profoundly anaemic; there was some oedema of the feet. He looked as if he were suffering from pernicious anaemia or advanced malignant disease. On February 25th, 1895, when I first saw this patient, the red corpuscles numbered $2,400,000$ per cubic millimetre; the haemoglobin was 34 per cent. The red corpuscles were perfectly normal in size and shape; there was no poikilocytosis. The white corpuscles were not markedly increased in number, and were normal in character." Whether in the later stages of this case the blood picture changed to that of pernicious anaemia, as it does in the later stages of some cases of sprue, I am unable to say, for the blood was not again examined.

This patient died on February 23rd, 1896. The post-mortem examination was made by Professor Robert Muir. The body was extremely emaciated and the muscular atrophy profound; it exactly resembled, both during life and after death, the extreme muscular wasting which is seen in advanced cases of myopathic muscular atrophy. Remarkable changes, which we have pictured in the published report of the case, were found in the muscles. In that paper" we state: "These muscular changes have not, so far as we are aware, been previously described; but we venture to think they will probably be found in other cases of the disease in which the muscles are, as they were in this case, extremely atrophied.

"In both of the cases which we have had the opportunity of studying, the voluntary muscles throughout the body were atrophied in quite a remarkable degree. During life the con dition exactly resembled that characteristic of the advanced stage of myopathic muscular atrophy. In neither of the cases was there any paralysis, but merely profound muscular weakness

${ }^{1}$ A Remarkable and Hitherto Undescribed Muscular Lesion occurring in Sprue, with notes of a case of peculiar (? Myopathic) Muscular Atrophy in which somewhat similar changes were present. With Pathological Report vol. vi, i. 5 . .) proportionate to the muscular atrophy; there were no fibrillary twitchings; the electrical changes were not noted.

"In the first case, in which we were able to make a complete post-mortem examination, the peripheral nerves were normal in every respect; in the second case the peripheral nerves were not examined.

"The portions of the voluntary muscles which were submitted to microscopical examination showed, in both cases, similar changes. These changes were much more marked in the first than in the second case. They consisted of great proliferation of the nuclei of the sarcolemma, which in places were enormously enlarged, and degenerative changes in the muscular fibres. In places, the muscular fibres had apparently been completely absorbed by the enlarged nuclei, the degenerated remains representing the remains of muscular fibres. In the second case there appeared to be some increase of the intermuscular connective tissue. This seemed to be the result of the muscular atrophy and not its cause. There was no lipomatosis.

"It is probable, we think, that the muscular changes were due to the action of some toxin absorbed from the intestine."

Exactly similar muscular changes were found in a second case of sprue, in which the same extreme muscular atrophy was present, which came under my notice in May, 1899 . In that case the muscles were also examined by Professor Robert Muir.

I refer to these remarkable muscle changes in the hope that someone who has the opportunity of examining cases of sprue may make a microscopic examination of the muscles, or, better still perhaps, send specimens of the muscle removed after death to Professor Robert Muir, Glasyow University who will, I am sure, be glad to investigate further cases in the hope of throwing light on this very remarkable condition.

In a third case of profound muscular atrophy which occurred in a man, aged 64 , who came under my notice in September, 1896, very similar changes were found in the muscles by Professor Robert Muir. This was not a case of sprue, but the patient had been addicted for several years to chewing tobacco in great excess; his wife stated that he was hardly ever without a quid in his mouth. The patient himself told me that he never swallowed the saliva while " chewing"; he further stated that he had always been very susceptible to the action of tobacco; he had never in his life been able to smoke two pipes straight off; for some months before I saw him he had discontinued "chewing". in such large quantities. The only possible cause of the profound muscular atrophy and muscle changes in this case appeared to be nicotine poisoning.

My chief object in writing this note, however, is to suggest that in cases of Addison's (pernicious) anaemia a trial should be made of parathyroid extract. This idea occurred to me after reading Dr. H. H. Scott's paper in the BRITIsH Medical Journal of December 15th, 1923, in which he recorded some remarkable results obtained in sprue from parathyroid treatment. A few days after I had read Dr. Scott's paper I saw, in consultation with Dr. Ballantyne of Dalkeith, a case of Addison's (pernicious) anaemia, and advised him to try parathyroid extract together with arsenic. Unfortunately the patient was practically moribund when I saw her; she died two days afterwards and there was no chance of proving whether parathyroid treatment was likely to be successful in pernicious anaemia or not. I suggest, however, that it should be given a thorough trial.

A word in conclusion as to nomenclature. I have always thought that it is a mistake to name diseases after their discoverers, and in this case, since Addison's name is already associated with the tuberculous disease of the suprarenal capsules, the term "Addison's anaemia" is, I think, particularly unfortunate. A name should, if possible, indicate the cause of the disease, or, if that is not known, some of its important characteristics. Though the disease is in rare cases recovered from, and in many cases, under modern methods of treatment, is of longer duration than it was when Biemer gave the name "pernicious" to it, the term still, I think, is a good one, since it correctly differentiates it from other forms of apparently primary anaemia, such as chlorosis, the exact cause of which we do not know. Some years ago I suggested that if the term "pernicious" is thought undesirable, the disease might, until its exact cause is discovered, be termed gastro-intestinal, or perhaps gastro-intestinal-hepatic anaemia. 